\title{
Florence Bouchet, Le discours sur la lecture en France aux $\mathrm{XIV}^{\mathrm{e}}$ et $\mathrm{XV} \mathrm{V}^{\mathrm{e}}$ siècles: pratiques, poétique, imaginaire
}

\author{
Maria Colombo Timelli
}

\section{OpenEdition}

\section{Journals}

\section{Édition électronique}

URL : http://journals.openedition.org/studifrancesi/7477

DOI : 10.4000/studifrancesi.7477

ISSN : 2427-5856

\section{Éditeur}

Rosenberg \& Sellier

\section{Édition imprimée}

Date de publication : 1 décembre 2009

Pagination : 603-604

ISSN : 0039-2944

\section{Référence électronique}

Maria Colombo Timelli, «Florence Bouchet, Le discours sur la lecture en France aux xIV et xv siècles: pratiques, poétique, imaginaire », Studi Francesi [En ligne], 159 (LIII | III) | 2009, mis en ligne le 30 novembre 2015, consulté le 09 janvier 2021. URL : http://journals.openedition.org/studifrancesi/7477 ; DOI : https://doi.org/10.4000/studifrancesi.7477

Ce document a été généré automatiquement le 9 janvier 2021.

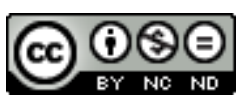

Studi Francesi è distribuita con Licenza Creative Commons Attribuzione - Non commerciale - Non opere derivate 4.0 Internazionale. 


\title{
Florence Bouchet, Le discours sur la lecture en France aux XIV et $\mathrm{XV}^{\mathrm{e}}$ siècles: pratiques, poétique, imaginaire
}

\author{
Maria Colombo Timelli
}

\section{RÉFÉRENCE}

FLORENCE BOUCHET, Le discours sur la lecture en France aux XIV et XV siècles: pratiques, poétique, imaginaire, Paris, Honoré Champion, 2008, pp. 392.

1 Dans l'histoire de la lecture, comme dans la plupart des domaines du savoir, les deux siècles envisagés dans ce beau livre de Florence Bouchet représentent une période de transition, d'autant plus significative qu'une véritable révolution s'y situe, avec l'invention et la diffusion de l'imprimerie.

2 Cette réflexion à plusieurs facettes part d'un constat: la complexité d'une situation où l'ancienne pratique de lecture à haute voix coexiste avec une lecture visuelle, comme le prouvent les nombreuses attestations des doublets «oïr et lire» dans les textes en vers et en prose, ainsi que les témoignages des historiens à propos des habitudes des grands seigneurs (Olivier de la Marche pour Charles le Téméraire entre autres); cependant, l'évolution des pratiques vers la lecture silencieuse est témoignée dans les manuscrits plus tardifs. F.B. ne néglige évidemment pas la part de la rhétorique ni le jeu des formules servant parfois uniquement à maintenir le contact entre auteur et destinataires: dans un tel cadre, le verbe «entendre», au sens double, auditif et intellectif, assume évidemment une importance majeure (chapitre 1, Lecteurs ou auditeurs?, pp. 23-55).

Oscillant entre difficulté et plaisir, entre les deux pôles du placere et du docere, la lecture n'est pas une activité neutre sur le plan moral; elle offre un plaisir capable de porter remède à la mélancolie et à l'ennui, et procure un profit moral qui s'exprime aussi à travers la dimension «spéculaire»; surtout, comme le soulignent nombre de prologues, 
elle porte à éviter «oiseuse», ce qui permet de contrecarrer le dénigrement de la littérature profane (chapitre 2, Plaisir et profit du lecteur, pp. 57-95).

4 Le chapitre 3 (L'écriture au service de la lecture, pp. 97-135) est consacré aux choix stylistiques et procédés d'écriture visant à favoriser la communication avec le lecteur: choix de la prose, pratique de la traduction (de l'ancien français, avec les adaptations en prose, du latin, voire traductions fictives dans le cas de certains romans), insertion de gloses, constituent autant de moyens destinés à promouvoir la lecture et à impliquer le lecteur dans la démarche herméneutique.

Une métaphore filée construite sur le monde végétal sous-tend le chapitre suivant (4, Une esthétique de la cueillette, pp. 137-168): l'auteur cueille son savoir dans des livres plus anciens, il recueille (autrement dit, il compile) de sources nombreuses et diverses; de son coté, le lecteur peut lui aussi procéder à une cueillette, à savoir à une lecture sélective ou consultative que facilitent les procédés divers de la mise en page (titres de chapitre, titre courants, tables, indices), technique qui se fait évidemment encore plus visible dans les anthologies, florilèges et recueils.

6 La lecture peut aussi faire l'objet d'une mise en fiction, que F.B. analyse selon quatre axes: mise en scène de l'auteur et du lecteur; analogie lecture / voyage; modèles du bon (ou mauvais) lecteur; analogie cimetière / bibliothèque, tombeau / livre en tant que lieu(x) de mémoire (chapitre 5, Lector in fabula, pp. 169-209).

7 Il est indéniable que le lecteur participe désormais à l'élaboration du sens du texte; c'est ce qui impose de prendre en compte certaines expressions trop souvent liquidées sous l'étiquette de «clichés», «formules» plus ou moins stéréotypées, et de les interroger directement: éloge du commanditaire / dédicataire, premier lecteur de l'œuvre, mais aussi appels au lecteur capable de corriger / compléter, recherche de collaboration et de connivence, formules phatiques pour assurer / garder le contact, tous ces passages se situant non seulement dans les prologues, mais dans l'ensemble des textes considérés (chapitre 6, L'autorité du lecteur, pp. 211-238).

8 Autre aspect caractéristique de cette littérature du Moyen Âge «tardif», quelques textes deviennent la source de controverses et querelles, diatribes diverses, correspondances poétiques (du Roman de la Rose à la Belle Dame sans merci), certains poèmes sollicitant même des réponses. Le rapport entre lecture et écriture se fait encore plus strict, interrogation et contradiction devenant des facteurs de création littéraire (chapitre 7 , La littérature, lieu et enjeu de débat, pp. 239-275).

9 Un ouvrage de ce genre ne peut évidemment pas laisser de côté l'objet sur lequel la lecture se fonde: le livre, vecteur de l'échange entre auteur et lecteur(s) (chapitre 8, Le lecteur au contact du livre, pp. 277-307). S'il est toujours regrettable d'oublier la matérialité du volume, sa transformation entre $\mathrm{XIV}^{\mathrm{e}}$ et $\mathrm{XV}^{\mathrm{e}}$ siècle, avec le passage graduel du manuscrit à l'imprimé, rend une telle réflexion absolument incontournable. F.B. souligne certains aspects marquants de cette évolution: du format à la mise en page, des tables des matières aux renvois internes, de l'iconographie à l'alternance des encres, des jeux linguistiques (acrostiches ou anagrammes, qui établissent une autre sorte de jeu entre auteur et lecteur) à la visualisation des textes poursuivie surtout par les Grands Rhétoriqueurs.

10 Les pages conclusives, qui portent un titre sans doute volontairement outré (L'invention $d u$ lecteur, pp. 309-323), synthétisent clairement les étapes fondamentales du parcours proposé: l'importance des prologues d'abord, qui révèlent, derrière la topique, 
l'importance du rapport qui s'instaure désormais entre auteur et lecteur(s); le glissement progressif, qui se complète entre $\mathrm{XIV}^{\mathrm{e}}$ et $\mathrm{Xv}^{\mathrm{e}}$ siècle, de la lecture orale, collective, vers la lecture oculaire, personnelle; l'implication de plus en plus importante des lecteurs à l'intérieur de l'œuvre; la fonction de l'imprimé, qui provoque l'entrée en scène de l'imprimeur-libraire dans le circuit de production et diffusion du livre, avec continuation de certains éléments, mais aussi rupture vers plus de liberté / responsabilité dévolue au lecteur quant à l'interprétation du contenu.

11 Dans le sillage des études célèbres de Jacqueline Cerquiglini-Toulet, ce livre s'appuie souvent sur des textes bien connus, mais a le mérite indéniable d'y porter un regard frais et pénétrant et d'offrir des réflexions clairement exposées, qui évitent soigneusement tout jargon appuyé.

Il est complété par quelques annexes: des prologues, épilogues, extraits tant de textes manuscrits que d'imprimés, particulièrement représentatifs des phénomènes étudiés (12 fragments au total, pp. 325-343). La bibliographie est présentée sous forme raisonnée: sources primaires (pp. 345-352; remarquons à ce propos que quelques éditions critiques récentes sont oubliées: il en est ainsi pour le Pèlerinage de Vie Humaine de Guillaume de Digulleville, édité par Frédéric Duval en 2006, pour le Guillaume d'Orange en prose, publié en trois volumes par Madeleine Tyssens, Nadine Henrard, Louis Gemenne, en 2000-2006, ou encore pour Paris et Vienne, édité par Annamaria Babbi en 1992) et sources secondaires (pp. 353, en trois sous-sections). Suivent l'Index des noms et titres (pp. 373-385), complément très utile, et un Index (évidemment sélectif) des notions (pp. 387-389).

Ouvrage très appréciable, qui fournit une synthèse importante sur l'évolution d'une pratique certes encore réservée à une minorité, mais à une minorité qui est en train de s'élargir, on nous pardonnera si nous lui réservons deux petites remarques: le corpus ne faisant pas l'objet d'une présentation, le lecteur ne découvre qu'au fur et à mesure les textes étudiés; surtout, mais cela ne saurait aucunement être reproché à l'auteur, l'absence de toute reproduction, même en noir et blanc: malgré les efforts déployés par F.B., il est extrêmement difficile de «montrer» par des mots, par exemple, les pages de titre des incunables ou des imprimés anciens, et encore davantage la mise en page de certains mss. Cela ne constitue sans doute pas un obstacle pour les lecteurs mieux informés, pour lesquels une simple allusion suffit (cf. par exemple la description de la page de titre du Perceforest de Galliot du Pré, p. 317), mais empêchera les lecteurs moins experts - je pense aux étudiants et aux jeunes chercheurs, qui pourront cependant profiter largement de ces pages - de se rendre compte pleinement de certains aspects visuels. 